\title{
Sentiment of App with Word Vectors
}

\author{
Preethi Kulkarni, C.V.P.R.Prasad
}

\begin{abstract}
Vector representations for language have been shown to be useful in a number of Natural Language Processing tasks. In this paper, we aim to investigate the effectiveness of word vector representations for the problem of Sentiment Analysis. In particular, we target three sub-tasks namely sentiment words extraction, polarity of sentiment words detection, and text sentiment prediction. We investigate the effectiveness of vector representations over different text data and evaluate the quality of domain-dependent vectors. Vector representations has been used to compute various vector-based features and conduct systematically experiments to demonstrate their effectiveness. Using simple vector based features can achieve better results for text sentiment analysis of APP.
\end{abstract}

\section{INTRODUCTION}

With a data blast driven by client created Internet content, the undertaking of handling colossal amounts of data accessible online is a considerable one past the abilities of human preparing. For example, motion picture merchants and fans are progressively intrigued by open assumptions with respect to new films. Entrepreneurs and clients are additionally increasingly anxious to find how individuals see new items.

Be that as it may, with the immense measure of data accessible web based, gathering and totaling film and item audits is a testing undertaking and machines must be used to assist analysts with information accumulation. One such methodology is that of slant examination, which has demonstrated famous throughout the years.

Opinion examination is a "bag" investigate issue that requires handling numerous NLP sub-undertakings, including angle extraction, subjectivity discovery, idea extraction, named substance acknowledgment and mockery location yet in addition reciprocal errands, for example, identity acknowledgment, client profiling and multimodal combination. Much work has been done on English notion investigation, however there is absence of research in the Chinese dialect field. Luckily, more analysts have begun to lead Chinese notion order in the most recent decade.

There are two primary ways to deal with Chinese estimation investigation inquire about: the monolingual methodology and the bilingual methodology. The previous spotlights on performing common slant examination assignments, for example, extremity identification, specifically dependent on Chinese dialect. The last influences on existing English dialect assets and machine interpretation methods to deal with Chinese normal dialect content.

Chinese estimation order, nonetheless, has certain attributes that vary from English slant arrangement. The most outstanding component of Chinese is the absence of between word dispersing, as a string of Chinese content is comprised

Revised Manuscript Received on September 22, 2019

Ms.Preethi, Asst. Professor, Department of Computer Science \& Engg, Malla Reddy Engineering College for Women, pradeepthi.preethigmail.com Dr. C.V.P.R.Prasad, Professor, Dept of CSE, Malla Reddy Engineering College for Women, prasadcvpr@gmail.com of similarly divided graphemes that are called characters. What's more, Chinese words frequently comprise of the mix of more than one Chinese character. In this way, it is important to fragment Chinese words previously breaking down the suppositions in Chinese writings. The above component prompts the second trademark that Chinese content is compositionally wealthy as far as semantics.

Since current Chinese characters develop from pictograms, they can be deteriorated into littler semantic mindful particles, named radicals. The third trademark is that Chinese has a moderately extraordinary or even inverse syntactic structure when contrasted with different dialects, particularly English, and techniques must be formulated to determine ambiguities present in Chinese syntactic parsing. For example, how linguistic structure trees vary in English and Chinese of an equivalent sentence. Whichever strategy is utilized, be it managed or unsupervised, a feeling dialect vocabulary is typically required for slant grouping.

Whatever is left of the paper is composed as pursues: Section "Issue Definition" clarifies issue definition and audit extent of this paper; Section "Development of Corpora and Lexica" presents later and present research on slant corpora and lexica; Section "Monolingual Approaches" portrays the distinctive perspectivesof feeling grouping techniques in Chinese dialect; Section "Multilingual Approach" talks about multi-lingual assessment arrangement strategies in Chinese language;Section "Testing Dataset" surveys some well known trial testing datasets and chose test results; at last, Section "End" closes the paper and proposes some future research headings.

\section{LITERATURE SURVEY}

Yang A, Lin J, Zhou Y. Method on Building Chinese Text Sentiment Lexicon[J]. Journal of Frontiers of Computer Science \& Technology, 2013.

A definitive objective of notion examination can be for the most part condensed as distinguishing conclusion or supposition marks of given writings. Contingent upon the kinds of conclusive mark, issues are normally partitioned into notion grouping and feeling/subjectivity recognizable proof. By the by, the two sub issues share comparable work process in accomplishing their last objectives. We condense the techniques and audit Chinese assumption investigation literary works on every one of the method.

Specifically, assumption assets like assessment lexica and corpora give the establishment everything being equal. Because of the shortage of Chinese feeling assets, making Chinese supposition assets is a noteworthy part of related research. Affected by the negative impact of space reliance and so as to spare human work, numerous works intend to create strategy that can make cross-area estimation asset in a semi-regulated way.

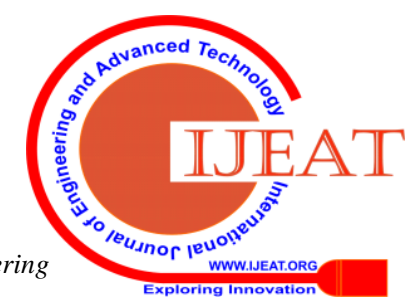




\section{Sentiment of App with Word Vectors}

With the slant asset, explore way bifurcates into machine learning based and information based strategies. Machine learning regards opinion arrangement as either a paired (positive or negative) or numerous class grouping issue. Customary machine learning goes for manual improvement of highlights that could recognize opinion of content out of various space information. Be that as it may, with an expanding pattern, late machine learning swings to building neural systems to learn skilled highlights consequently. Then, a few scientists spend energies on creating supposition classifiers that chip away at these highlights.

The other school which pursues the information based strategy examines dialect rules and linguistic or semantic relations. We audit the Chinese best in class in Section "Monolingual Approaches." Lastly, outlined by the generally various research led on English, another school of scientists uses a multilingual methodology which exchanges asset or strategy from the English research world to be utilized in Chinese circumstance.

Popescu A M, Etzioni O. Extracting Product Features and Opinions from Reviews[M]. Natural Language Processing and Text Mining. London: Spring, 2007:9-28. The key segments of OPINE depicted in this paper are the PMI include appraisal which prompts high-accuracy highlight extraction and the utilization of unwinding naming so as to locate the semantic introduction of potential conclusion words. The audit mining work most important to our exploration is that of (Hu and Liu, 2004) and (Kobayashi et al., 2004). Both recognize item includes from surveys, however OPINE altogether enhances both. (Hu and Liu, 2004) doesn't evaluate competitor highlights, so its accuracy is lower than OPINE's. (Kobayashi et al., 2004) utilizes an iterative self-loader approach which requires human contribution at each emphasis. Neither model unequivocally addresses composite (component of highlight) or certain highlights. Different frameworks (Morinaga et al., 2002; Kushal et al., 2003) additionally take a gander at Web item audits however they don't extricate suppositions about specific item includes. OPINE's utilization of meronymy lexico-syntactic examples is like that of numerous others, from (Berland and Charniak, 1999) to (Almuhareb and Poesio, 2004).

Perceiving the emotional character and extremity of words, expressions or sentences has been tended to by numerous creators, including (Turney, 2003; Riloff et al., 2003; Wiebe, 2000; Hatzivassiloglou and McKeown, 1997). Most as of late, (Takamura et al., 2005) provides details regarding the utilization of turn models to surmise the semantic introduction of words. The paper's worldwide streamlining approach and utilization of various wellsprings of imperatives on a word's semantic introduction is like our own, yet the instrument contrasts and they as of now overlook the utilization of syntactic data. Abstract expressions are utilized by (Turney, 2002; Pang and Vaithyanathan, 2002; Kushal et al., 2003; Kim and Hovy, 2004) and others so as to group surveys or sentences as positive or negative. Up until this point, OPINE's emphasis has been on separating and investigating conclusion phrases comparing to explicit highlights in explicit sentences, as opposed to on deciding sentence or survey extremity.

Zhang C G, Liu P Y, Zhu Z F, et al. A sentiment analysis method based on a polarity lexicon $[\mathrm{J}]$. Journal of Shandong University, 2012.
We look at the latest strategies for ascertaining the setting free vocabulary extremity and the methods for refreshing the extremity for explicit areas. We will break down their individual qualities and shortcomings.

In 2002, Turney presented a straightforward yet powerful unsupervised learning calculation, Pointwise Mutual Information and Information Retrieval (PMI-IR), to gauge the semantic closeness between two words. He expected that two words with comparable implications will in general seem together. So as to quantify the closeness of an explicit word to a reference word, he proposed to utilize the proportion between the two words' co-events and the chose word's event in the record set to assess their likeness. In 2006, Ye et al. adjusted the PMI-IR calculation to quantify the assumption extremity of Chinese words. They chose Excellent as the positive reference word and Poor as the negative reference word. These two words would make up a reference word combine (RWP). The assumption extremity of the word was processed dependent on the recurrence of its cooccurrence with each reference word in the Google output.

Thus, Zhu et al. likewise chose their own reference words for every feeling class. Be that as it may, Zhu et al. used a general lexicon HowNet to gauge the word's closeness to the reference word. In HowNet, a word may comprise of numerous meanings. Every implication may comprise of various sememes, the littlest unit to pass on an explicit significance. HowNet sorts out the words containing the samesememe together into a set and after that arranges these sememe sets into a tree of hyponyms. Zhu et al. proposed to quantify a word's most limited way to every one of the assessment reference word in HowNet and contrasted the way length with ascertain the word's extremity.

The above methodologies can be abridged into the accompanying advances. Right off the bat, a reference word for every notion shaft is resolved. At that point, the outside corpus source is chosen. Ultimately, the extremity of any word is assessed dependent on the closeness association with these reference words in the chose corpus. These strategies are compelling in determining an extremity score for any word, however they are flexible to the progressions of content space. As talked about in the presentation, some broad clear words might be sure in one space yet turn negative in another. Thinking about this, few scientists have proposed the plans to refresh a word's extremity for explicit spaces, as portrayed in the accompanying section.

Vishnu et al. acquainted their technique with gather the Domain Independent Dictionary (DID) and Domain Specific Dictionary (DSD) from the SentiWordNet and area explicit corpus. Right off the bat, they chose a rundown of applicant words. At that point, they determined the area free extremity esteem (sw) for each word from SentiWordNet. The positive esteem spoke to positive assessment and negative esteem spoke to the inverse. A short time later, they gauged the distinction in the recurrence proportionality (dfp) of a word in positive and negative content information, which gave its space particular extremity. For every hopeful word, if its dfp and sw had a similar sign, it inferred that there was no extremity moving in this area. This word would be incorporated into DID and sw would be its extremity. 
On the off chance that sw and dfp had distinctive signs, the word would be incorporated into DSD and dfp would be its extremity esteem. A comparable methodology could likewise be connected to different areas. Every content dataset would create its very own DIDs and DSDs. The meeting some portion of all DIDs would give a rundown of general space free words and their comparing extremity esteems. DSD would create the rundown of area explicit words. So also, Demiroz et al. additionally estimated the space autonomous extremity from SentiWordNet and term recurrence distinction from the content corpus. On the off chance that the signs for a specific word couldn't help contradicting one another, they refreshed the word's extremity dependent on one of the accompanying four techniques:

Flip: Replace the original polarity with its opposite.

Objective Flip: Switch the original objective words to either positive or negative. Or, switch the original subjective word to objective

Shift: Shift the polarity of that word towards the other pole Delta Score: Compute the new polarity based on thedifference in term frequency

In addition, they additionally given distinctive criteria to decide the words for extremity refresh. For instance, refresh the best $\mathrm{k} \%$ of words that demonstrate a difference, refresh when contradiction surpasses a limit or iteratively refresh until the point when no enhancement can be made to the approval set. As should be obvious from the above thoughts, analysts essentially ascertain the area autonomous extremity for all words as a base and afterward create a slight alteration on the extremity of those words dependent on the given space explicit corpus. Nonetheless, the real issue referenced above still exists: their calculation's execution will to a great extent rely upon the adequacy of general assumption lexicon. Any inconsistency from the word reference may cause incredible mistakes in creating the area subordinate extremity. Thinking about this, we propose a calculation that does not depend on the general slant word reference. Rather, it depends absolutely on the word's dispersion in the content dataset.

Shi F, Fu Y, Feng Y, et al. Blog Sentiment Orientation Analysis Based on Dependency Parsing[J]. Journal of Computer Research \& Development, 2012, 49(11):2395-2406.

Researches and hot spots for Microblogging

Conventional content mining subject has been widely examined, but since of smaller scale blog with some auxiliary parts of the interpersonal organization data, the utilizing in its customary content mining calculations displaying isn't sufficiently exact. Standard related examination abroad incorporates Twitter investigation by $\mathrm{H}$. Kwak et al., seen in, and TwitterRank thought proposed by $\mathrm{J}$ Weng et al. in; and Domestic Zhang Chenyi et al. proposed a model dependent on the LDA produced Microblogging, and Microblogging subject assistant mining which can been found in; $\mathrm{Li} \mathrm{Si}$ proposed the LQE model and Bowen averaging calculation to extricate related blog, seen in. The above consequences of Microblogging explores are from numerous points, which furnish an essential reference to manage Microblogging in this paper.

Reliance Analysis

Reliance parsing is first proposed by L.Tesniere in, the center of which is spoken to by coupling the connection between words. During the 1970s, Robinson made four adages about reliance syntax reliance seen in, and because of the unique idea of the Chinese dialect structure, there is a fifth maxim seen in. Penn Chinese tree is a Chinese Structure Grammar Library seemed before and examined by more individuals, seen in, and ctbparser open source toolbox can naturally acquire the conditions between words in. As of now reliance parsing technique has been generally utilized, including: various leveled reliance parsing in, in light of investigation of passionate words related with the development of reliance and examination, which can be seen in, and other substance based dynamic learning Chinese reliance sentence structure. The above explores from various fields of reliance investigation were done on the content, which made huge commitments to direct Microblogging linguistic structure reliance examination in this paper.

\section{CONCLUSION}

Right now passionate data extraction strategies are broadly utilized in numerous fields, and there are two techniques, in particular corpus-based methodology and lexicon put together methodology with respect to the assessment of the words as far as extraction and segregation. Hatzivassiloglou et al. separated a great deal of modifiers assessment of the words from a huge corpus of Wall Street Journal in. Kobayashi et al. by means of the syntactic investigation (attributes, assessment words) acquired assessment of question in. Qiu and others utilize the conditions between word feeling and assessment of question acquire the assessment of protest in. Hou Min et al, from the Microblogging dialect highlights and conclusion investigation system, set forward perspectives sentence extraction methodology dependent on the recognizable proof and assessment of the passionate question word reference and expression semantic principles in. Ms Wang Qian, He Tingting separated content enthusiastic components from the expression subordinate investigation in. The above discoveries for content enthusiastic preparing give an essential premise to managing the passionate components extraction in this paper, which views Microblogging as the handling object. D. Related Work Introduction In past examinations, the proposed strategy related with reference and Both reference and direct enthusiastic word extension by the reliance parsing, where the technique for Qiu et al. can just broaden the passionate words which have an immediate reliance in, while the OWP calculation can at the same time expand the term with a direct enthusiastic relationship and circuitous conditions in, and the review rate tests of is superior to the technique in. Consequently, OWP technique is one of the key innovations to accomplish the objective of our paper. Reference positioned first and second in NLPCC2012 enthusiastic components removed assessment, which can be seen in. The principle techniques can be closed as pursues: based on enlisting the highlights of Microblogging dialect, it built the passionate expression word reference, decided the extremity of the sentence by the expression rules, concentrated on the negative shape, set up the theme based methodologies like OBJ frame, and finished the small scale Bo opinion examination. 


\section{Sentiment of App with Word Vectors}

Reliant way was led in this paper to achieve the programmed production of enthusiastic lexicon, and after that we did the Microblogging passionate factor extraction dependent on the reliance parsing. Like the article, reference took an interest in the COAE2008 assessment; notwithstanding, there are the accompanying contrasts: right off the bat, its protest is a content as opposed to managing the Microblogging; also, the test informational collection is little, just around 3000 sentences; thirdly, it embraced the experimentation assessment technique which you can generally change the advancement. Thought about reference, the protest in this paper is a Microblogging with handling informational index of 17500 small scale Bo and 32000 sentences; the outcomes were prepared with no reference answers to the control alteration; and assessment results were specifically submitted to the gathering and the general population.

\section{REFERENCES}

1. Zhao Y, Qin B, Liu T. Estimation Analysis[J]. Diary of Software,2010, 21(8):1834-1848.

2. Yang A, Lin J, Zhou Y. Strategy on Building Chinese Text Sentiment Lexicon[J]. Diary of Frontiers of Computer Science and Technology, 2013.

3. Popescu A M, Etzioni O. Removing Product Features and Opinions from Reviews[M]. Common Language Processing and Text Mining. London: Spring, 2007:9-28.

4. Yao Tianfang, Lou Decheng. A supposition digging framework for Chinese car surveys [J]. Diary of Computer Applications. 2006.

5. Zhao Y, Qin B, Che W X, et al. Examination Expression Recognition Based on Syntactic Path[J]. Diary of Software, 2011, 22(5):887-898.

6. Zhang C G, Liu P Y, Zhu Z F, et al. An estimation examination strategy dependent on an extremity dictionary [J]. Diary of Shandong University, 2012, 47(3):47-50.

7. Shi F, Fu Y, Feng Y, et al. Blog Sentiment Orientation Analysis Based on Dependency Parsing[J]. Diary of Computer Research and Development, 2012, 49(11):2395-2406.

8. Zhang Shan, Yu Liubao, Hu Changjun. Estimation examination of Chinese Mircro-blog dependent on feelings and enthusiastic words [J]. Software engineering, 2012, 39(11A): 146-148, 176.

9. Xie L, Zhou M, Sun M. Various leveled Structure Based Hybrid Approach to Sentiment Analysis of Chinese Micro Blog and Its Feature Extraction [J]. Diary of Chinese Information Processing, 2012, 26(1):73-83.

10. Sun J, Xueqiang L, Zhang L. On estimation examination of Chinese microblogging dependent on vocabulary and machine learning. [J]. PC Applications and Software, 2014. 